\title{
Proteomic analysis of porcine mesenchymal stem cells derived from bone marrow and umbilical cord: implication of the proteins involved in the higher migration capability of bone marrow mesenchymal stem cells
}

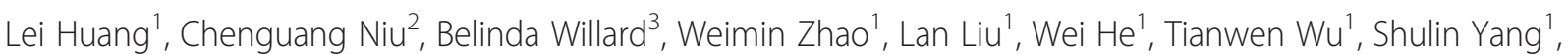
Shutang Feng ${ }^{1}$, Yulian $\mathrm{Mu}^{1^{*}}$, Lemin Zheng ${ }^{2^{*}}$ and Kui $\mathrm{Li}^{1^{*}}$

\begin{abstract}
Introduction: Mesenchymal stem cells (MSCS) have the ability to proliferate in vivo with a large variety of differentiation potentials and therefore are widely used as an ideal material for cell therapy. MSCs derived from pig and human sources are similar in many aspects, such as cell immunophenotype and functional characteristics. However, differences in proteomics and the molecular mechanisms of cell functions between porcine bone marrow MSCs (BM-MSCs) and umbilical cord MSCs (UC-MSCS) are largely unknown. To the best of our knowledge, MSCs collected from different tissue have specific phenotype and differentiation ability in response to microenvironment, known as a niche.

Methods: Porcine BM-MSCs and UC-MSCs were evaluated with flow cytometric and adipogenic and osteogenic differentiation analyses. We used isobaric tagging for relative and absolute quantitation (iTRAQ), combined with liquid chromatography-tandem mass spectrometry, to identify differentially expressed proteins (DEPs) between these two types of MSCs. Kyoto Encyclopedia of Genes and Genomes pathway and phenotype analyses were used to understand the links between cell migration ability and DEPs.

Results: Two separate iTRAQ experiments were conducted, identifying 95 DEPs (95\% confidence interval). Five of these proteins were verified by Western blotting. These 95 DEPs were classified in terms of biological regulation, metabolic process, developmental process, immune system process, reproduction, death, growth, signaling, localization, response to stimulus, biological adhesion, and cellular component organization. Our study is the first to show results indicating that porcine BM-MSCs have a higher migration capability than UC-MSCs. Finally, one of the DEPs, Vimentin, was verified to have a positive role in MSC migration.

(Continued on next page)
\end{abstract}

\footnotetext{
*Correspondence: mouyulian@caas.cn; zheng|@bjmu.edu.cn; likui@caas.cn

${ }^{1}$ State Key Laboratory of Animal Nutrition and Key Laboratory of Farm

Animal Genetic Resources and Germplasm Innovation of Ministry of

Agriculture, Institute of Animal Sciences, Chinese Academy of Agricultural

Sciences, No. 2 Yuanmingyuan West Road, Haidian District 100193, Beijing,

China

${ }^{2}$ The Institute of Cardiovascular Sciences and Institute of Systems

Biomedicine, School of Basic Medical Sciences, and Key Laboratory of

Molecular Cardiovascular Sciences of Ministry of Education, Peking University

Health Science Center, No. 38 Xueyuan Road, Haidian District 100191, Beijing,

China

Full list of author information is available at the end of the article
}

C Biomed Central (c) 2015 Huang et al.; licensee BioMed Central. This is an Open Access article distributed under the terms of the Creative Commons Attribution License (http://creativecommons.org/licenses/by/4.0), which permits unrestricted use, distribution, and reproduction in any medium, provided the original work is properly credited. The Creative Commons Public Domain Dedication waiver (http://creativecommons.org/publicdomain/zero/1.0/) applies to the data made available in this article, unless otherwise stated. 
(Continued from previous page)

Conclusions: These results represent the first attempt to use proteomics specifically targeted to porcine MSCs of different tissues. The identified components should help reveal a variety of tissue-specific functions in tissue-derived MSC populations and could serve as important tools for the regeneration of particular tissues in future stem cell-based tissue engineering studies using animal models.

\section{Introduction}

Mesenchymal stem cells (MSCs), which are a type of adult stem cell developed from the mesoderm, can be isolated from the brain, liver, lung, kidney, fat, bone marrow, peripheral blood, umbilical cord blood, umbilical cord, placenta, amniotic fluid, and other tissues [1]. MSCs possess the potential for self-renewal and pluripotency and play an important role in tissue repair and regeneration [2]. When cultured in vitro, MSCs exhibit strong proliferation and can be induced to differentiate into bone, cartilage, adipogenic, and myogenic tissue; therefore, MSCs are widely used for bone and muscle repair in pre-clinical evaluation and clinical studies [1]. In addition, extensive clinical studies on MSC-based therapies have been conducted on many human diseases, including chronic graft-versus-host disease, systemic lupus erythematosus, cirrhosis, diabetes, acute kidney injury, and a variety of neurodegenerative diseases [1]. Bone marrow MSCs (BM-MSCs) are an important source of the adult stem cells widely used in basic and clinical research [3]. However, these cells are limited by the inconvenience of sample collection and by a reduced proliferation and differentiation capacity, coupled with effects of the donor's age [2]. Therefore, searching for the detailed mechanism underlying these phenotypes and eliminating these limitations are important. In contrast, some studies have shown that umbilical cord MSCs (UC-MSCs) are similar to BM-MSCs regarding cell surface markers, physiological characteristics, proliferation and differentiation characteristics, and protein expression spectra; therefore, similar to BM-MSCs, UCMSCs may be used for allogeneic stem cell transplantation in cell regeneration therapy [4]. MSCs that reside in different microenvironments have many similarities; however, a detailed comparison between BM-MSCs and UC-MSCs is required to verify their potential for use in clinical therapy.

In recent years, miniature pigs have been widely used as an animal model in stem cell biology, which is important for the treatment of human diseases and for xenotransplantation. Because the organ size, physiological level, genetic characteristics, and other aspects of the pig model are similar to those of humans, the findings on porcine stem cells may provide a theoretical basis and practical guidance for clinical applications in human diseases [5]. Therefore, it may be possible to use pig-derived stem cells as an alternative resource to treat human diseases. However, some researchers have shown that different sources of MSCs exhibited differences in gene expression patterns under specific culture conditions [3,6]. In addition, these cells could produce specific organizational structures depending on their growth environment and on their response to different ectopic microenvironments, thereby differentiating into different cell types [7]. Therefore, it is important to understand the molecular basis of these features and signaling mechanisms of MSCs for the clinical application of cell therapy. Analyzing gene expression levels and taking advantage of chip or sequencing technology would help us to understand the molecular mechanism of cellular functions; however, transcript level information may not completely overlap with protein levels [8]. Therefore, proteomic research would aid in understanding the molecular mechanism of MSC differentiation [6]. Compared with transcriptome data, proteome analysis could be used to study many aspects of the proteome, including protein expression levels, protein stability, subcellular localization, post-translational modifications, and protein interactions [8]. However, there are some limitations to proteomic approaches based on two-dimensional electrophoresis and mass spectrometry (MS) techniques. For example, detecting hydrophobic proteins and phosphorylated proteins is difficult [9]. These limitations have driven the development of new technologies for protein identification and quantitation. iTRAQ (isobaric tagging for relative and absolute quantitation) is a gel-free technique used in quantitative proteomic analysis that can identify lowabundance proteins [9]. This type of proteomic analysis may help researchers to understand the proteomic differences of diverse sources of MSCs.

A proteomic analysis of MSCs was first reported in 2001 [6]. Since then, MS-based proteomic technology has increasingly become an effective tool for detecting differentially expressed proteins (DEPs) of MSCs derived from various sources or in the state of in vitro differentiation [10]. Researchers have used proteomic technology to identify DEPs of human MSCs in the process of osteogenic differentiation [11]. In another study, rabbit BM-MSCs were induced by 5 -azacytidine (5-aza) to differentiate into myocardial cells, and the resulting proteomic changes were analyzed [12]. Welsh et al. identified 
these up- and down-regulated proteins during adipogenic differentiation of MSCs [13]. However, MSCs derived from various sources exhibit differences in their protein expression profiles [6]. A proteomic comparison between BM-MSCs and UC-MSCs has already been reported for humans but not for large animals, such as pigs. Neng Seng et al. conducted a comparative analysis of the proteome of BM-MSCs in different generations isolated from Chinese miniature pigs only [14], and their research did not include a comparison of MSCs derived from different tissues. In the study of human MSCs, Guo Li et al. compared the proteome of BM-MSCs and UC-MSCs and then studied different proteins associated with cell migration [2]. Given the limitation in collecting human specimens, their experiment used a two-to-two design, and the two-dimensional electrophoresis-based MS and tandem MS (MS/MS) analysis detected only six DEPs between these two types of MSCs. Therefore, it is important to use an animal model, such as the pigs described above, and sensitive proteomic detection to uncover the differences between MSCs. In this study, we compared the DEPs from match-paired BM-MSC and UC-MSC samples, which were collected from the same piglets and cultured under identical conditions, and found 95 DEPs involved in different aspects of cell biology, especially cell motility. Additionally, iTRAQ quantitative proteomic techniques were used to clarify DEPs between BM-MSCs and UC-MSCs obtained from Wuzhishan mini-pigs. Accuracy of the results was assessed by Western blot. The results from these experiments indicate that the protein expression profiles of these two types of MSCs such as Vimentin, LGALS3, and TMSB4X are largely distinguishable in terms of cell motility.

\section{Methods}

\section{Animals}

The Animal Care Committee of the Chinese Academy of Agricultural Sciences approved all animal procedures. The newborn pigs were purchased from the National Germplasm Resources Center of Laboratory Miniature Pig.

\section{Isolation and culture of porcine mesenchymal stem cells}

The MSCs were isolated from porcine umbilical cord (UC-MSCs) and bone marrow (BM-MSCs) as described previously. Briefly, the umbilical cords were collected when the piglets were born, and intact femurs were harvested from the same four Wuzhishan inbred pigs (WZSP) (Institute of Animal Sciences, Chinese Academy of Agricultural Sciences, Beijing, China) by sterile operation at 42 days after birth. The umbilical cord tissue was diced into 2- to 3- $\mathrm{mm}^{3}$ pieces, and the MSCs were separated by using a substrate-attached explant method. The bone marrow stem cells were extracted and centrifuged at
$200 \mathrm{~g}$ for 5 minutes. The isolated MSCs were cultured in Dulbecco's modified Eagle's medium/F12 (DMEM/F12) (12500; Gibco, part of Life Technologies, Carlsbad, CA, USA) medium with $20 \%$ ( $\mathrm{vol} / \mathrm{vol}$ ) fetal bovine serum (10099; Gibco), 50 units/mL penicillin G, and $50 \mu \mathrm{g} / \mathrm{mL}$ streptomycin and incubated at $37^{\circ} \mathrm{C}$ under $5 \%(\mathrm{vol} / \mathrm{vol})$ $\mathrm{CO}_{2}$ in $100 \%$ humidified air. The media were changed every other day. The MSCs were harvested by digestion with $0.05 \%$ (wt/vol) trypsin-EDTA (25300054; Gibco) when the rate of cell fusion reached $80 \%$. Cells were replanted in 100-mm dishes at a density of $1 \times 10^{4} / \mathrm{cm}^{2}$.

\section{The evaluation of mesenchymal stem cells by flow cytometric analysis}

The cultured MSCs were digested with $0.05 \%$ (wt/vol) trypsin-EDTA (Gibco), followed by washing with cold autoMACS Rinsing Solution $\left(2^{\circ} \mathrm{C}\right.$ to $8^{\circ} \mathrm{C}$; Miltenyi Biotec, Bergisch Gladbach, Germany) three times. The pellets were resuspended in $1 \%(\mathrm{wt} / \mathrm{vol})$ bovine serum albumin (Sigma-Aldrich, St. Louis, MO, USA) for $30 \mathrm{mi}-$ nutes at $4^{\circ} \mathrm{C}$ to block non-specific binding. Then, the UC-MSCs were incubated with rat anti-mouse CD31APC (PECAM-1) (Miltenyi Biotec), mouse anti-human CD34-PE (Miltenyi Biotec), mouse anti-human CD45-PE (Miltenyi Biotec), or mouse anti-human CD90-FITC (Thy-1) (Abcam, Cambridge, MA, USA) monoclonal antibodies at $4^{\circ} \mathrm{C}$ for 30 minutes, respectively. The BMMSCs were incubated with mouse anti-human CD29FITC (Miltenyi Biotec), mouse anti-human CD34-PE (Miltenyi Biotec), rat anti-mouse CD44-FITC (Miltenyi Biotec), mouse anti-human CD45-PE (Miltenyi Biotec), or mouse anti-human CD90-FITC (Thy-1) (Abcam) monoclonal antibodies at $4^{\circ} \mathrm{C}$ for 30 minutes, respectively. The flow cytometric acquisition and data analysis were performed by using a BD FACSCalibur flow cytometer and Cell Quest software (BD Biosciences, San Jose, CA, USA). As a negative control, cells were incubated only with the corresponding isotype antibody, including rat IgG2a-APC (used for CD31; Miltenyi Biotec), mouse IgG2a-PE (used for CD34 and CD45; Miltenyi Biotec), rat IgG2b-FITC (used for CD44; Miltenyi Biotec), and mouse IgG1-FITC (used for CD29 and CD90; Miltenyi Biotec). These specimens could be placed in 4\% paraformaldehyde for short-term preservation. Three independent flow cytometric experiments were performed.

\section{Adipogenic and osteogenic differentiation of mesenchymal stem cells}

To evaluate MSC abilities, adipogenic and osteogenic differentiation assays were performed on isolated cells. Osteogenesis differentiation medium (Gibco) or adipogenesis differentiation medium (Gibco) was added into a culture when the fusion rate reached approximately $80 \%$. The cells were cultured at $37^{\circ} \mathrm{C}$ in $5 \%(\mathrm{vol} / \mathrm{vol}) \mathrm{CO}_{2}$ in 
$100 \%$ humidified air. The media were changed every 3 days, and the cells were cultured for 2 to 3 weeks before collection. Then, Alizarin Red S staining was used to analyze osteogenic lineages, whereas Oil Red $\mathrm{O}$ was used to analyze lipid droplets. Adipogenic and osteogenic differentiation assays were conducted three times for all four donor cells.

\section{Trypsin digestion and ITRAQ labeling}

All the reagents and buffers required for iTRAQ labeling and cleaning were purchased from Applied Biosystems (Foster City, CA, USA). The iTRAQ labeling assay was conducted in accordance with the instructions of the manufacturer. Briefly, after digestion in culture flasks, total MSCs were collected and washed with cold phosphate-buffered saline (PBS) three times $\left(2^{\circ} \mathrm{C}\right.$ to $\left.8^{\circ} \mathrm{C}\right)$. Cell pellets were directly used for extracting proteins or frozen in $-80^{\circ} \mathrm{C}$. The protein extraction process was carried out on ice in cold lysis buffer containing complete protease inhibitor cocktail (Roche, Basel, Switzerland). Proteins were stored in $-80^{\circ} \mathrm{C}$. Repeated freeze-thaw cycles were avoided. The proteins were dissolved in $8 \mathrm{M}$ urea supplemented with $10 \mathrm{mM}$ DTT, pH 8.5 (Amesco, St. Louis, MO, USA), and protein concentrations were determined by using the Bradford assay. Proteins were dissolved, denatured, alkylated, and digested with trypsin (Sigma-Aldrich; $1: 20$, wt $/ \mathrm{wt}, 37^{\circ} \mathrm{C}$ for 18 hours). To label peptides with the iTRAQ reagent, 1 unit of label (defined as the amount of reagent required to label $100 \mu \mathrm{g}$ of protein) was thawed and reconstituted in $70 \mu \mathrm{L}$ of ethanol. The digestion reactions from UC-MSCs and from BMMSCs were separately labeled with the 114 and 117 iTRAQ reagents, respectively. To identify more proteins, a strong cation exchange column (Applied Biosystems) was used to separate the mixed peptides. The elution buffers used were elution buffer A, containing $5 \mathrm{mM}$

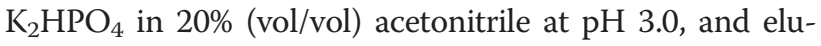
tion buffer B, containing $5 \mathrm{mM} \mathrm{K}_{2} \mathrm{HPO}_{4}$ in $20 \%$ (vol/vol) acetonitrile and $350 \mathrm{mM} \mathrm{KCl}$ at $\mathrm{pH}$ 3.0. The labeled peptides were reconstituted in phase $\mathrm{A}$ and injected at a flow rate of $0.7 \mathrm{~mL} /$ minute into a high-resolution strong cation exchange (SCX) column $(4.6 \times 250 \mathrm{~mm} 5 \mu \mathrm{m}$; Thermo BioBasic, USA). After loading, the SCX column and C 18 precolumn were flushed with a three-step gradient sodium chloride solution $(0,50$, and $100 \mathrm{mM})$ for 66 minutes. Then, the elution of the cation exchange groups was performed with an Agilent 1100 series high-performance liquid chromatography (HPLC) system, which was equipped with an autosampler, a 2/6 valve, and diode array detector $(220 \mathrm{~nm})$ (Agilent, Waldbronn, Germany), and 35 fractions were collected. Before liquid chromatography-MS/MS (LC-MS/MS), each fraction was desalted by using an SP-10 precolumn.

\section{Analysis by triple quadrupole time-of-flight tandem mass spectrometer}

The eluted fractions were delivered into a nano reversed phase column (5- $\mu \mathrm{m}$ Hypersil C18 column, $75 \mu \mathrm{m} \times$ $150 \mathrm{~mm}$; Thermo Fisher Scientific, Waltham, MA, USA) mounted in a Prominence Nano HPLC system (Shimadzu, Nakagyo-ku, Kyoto, Japan) and were eluted with an acetonitrile gradient from $5 \%$ to $40 \%$ containing $0.1 \%$ formic acid for 75 minutes at $400 \mathrm{~nL}$ per minute. The eluates were directly entered into a triple quadrupole time-of-flight (TOF) 5600 System (AB Sciex, Concord, ON, Canada), which was fitted with a Nanospray III source (AB Sciex) and with a pulled quartz tip as the emitter (New Objectives, Woburn, MA, USA), in positive ion mode and in a data-dependent manner, with full MS scan from 350 to $1,800 \mathrm{~m} / \mathrm{z}$.

The data were acquired by using an ion spray voltage of $2.5 \mathrm{kV}$, a curtain gas of 30 pounds per square inch (PSI), a nebulizer gas of 6 PSI, and an interface heater temperature of $150^{\circ} \mathrm{C}$. The MS was operated with a resolving power of 30,000 full width at half maximum (FWHM) for TOF-MS scans. For information-dependent acquisition, survey scans were acquired in 250 milliseconds, and as many as 20 product ion scans were collected when exceeding a threshold of 125 counts per second, with a +2 to +5 charge state. A rolling collision energy setting was applied to all precursor ions for collision-induced dissociation. Dynamic exclusion was set at half the peak width (approximately 8 seconds), and then the precursor was refreshed off the exclusion list.

\section{Database searching and protein quantitation}

In this study, we used ProteinPilot software 4.0 (AB Sciex, Foster City, CA, USA), including the Paragon ${ }^{\text {tw }}$ and Pro Group ${ }^{\mathrm{ma}}$ algorithms, to interpret raw data files produced by MS. The parameters for searching were as follows: iTRAQ four-plex peptide labeled, trypsin digestion with only 1 maximum missed cleavage, carbamidomethylation for cysteine residues, variable oxidation for methionine, quadrupole TOF electrospray ionization, and identification, focusing on biological modifications. The tolerances were specified as $\pm 0.05 \mathrm{Da}$ for peptides and $\pm 0.05 \mathrm{Da}$ for MS/MS fragments. The National Center for Biotechnology Information and Swiss-Prot protein databases were chosen for searching, and the false discovery rate was controlled at $1 \%$ by using the integrated tools in ProteinPilot software. For protein assembling, the Pro Group algorithm was used to find the smallest number of proteins that could explain all the fragmentation spectral evidence.

Protein quantification was also performed by using ProteinPilot software, which automatically calculated the relative abundance of iTRAQ-labeled peptides and their corresponding proteins. Corrections were made for the 
impurity of iTRAQ reagents on the basis of the data provided by the manufacturer. For other similar errors in analyses, iTRAQ ratios were normalized by autobias, which used all data to calculate the bias correction factor.

\section{Gene Ontology annotation and enrichment analysis} DEPs were annotated by using the Sus scrofa Gene Ontology (GO) annotations database (updated on Jan. 2, 2014). The GO enrichment analysis was based on GO databases and was conducted by using tools displayed on the GO website. All the databases and tools were downloaded or linked from the website [15].

\section{KEGG pathway analysis}

Pathway analyses of DEPs were based on the Kyoto Encyclopedia of Genes and Genomes (KEGG). The KEGG application programming interface and related databases were used to study the protein pathways. All of the resources were acquired from the website [16].

\section{Western blotting}

The cells were washed with ice-cold PBS and lysed in mammalian protein extraction reagent (Thermo Scientific, Waltham, MA, USA) containing a protease inhibitor mixture (Roche Applied Science, Indianapolis, IN, USA). Cell lysates were centrifuged at $12,000 \mathrm{~g}$ for $10 \mathrm{mi}$ nutes at $4^{\circ} \mathrm{C}$, and the supernatant was collected. The protein concentrations were measured by using the Bradford protein assay with a Bicinchoninic Acid Protein Assay Kit (Thermo Scientific). In total, $20 \mu \mathrm{g}$ of total protein was separated by $12 \%$ (wt/vol) SDS-PAGE and transferred onto a polyvinylidene fluoride (PVDF) membrane (Millipore, Billerica, MA, USA). Then, the membrane was blocked with $2 \%(\mathrm{wt} / \mathrm{vol})$ bovine serum albumin (BSA) (Sigma-Aldrich) for 1 hour. Next, the previously indicated primary antibodies were used to probe the membrane overnight at $4^{\circ} \mathrm{C}$. After extensive washing with Tris-buffered saline with Tween 20 (TBS-T), the membrane was incubated with secondary antibodies for 1 hour at room temperature. Bands were visualized by using SuperSignal West Pico Chemiluminescent Substrate (Pierce, part of Life Technologies) and recorded on $\mathrm{x}$-ray films (Fuji Medical, Tokyo, Japan). Finally, the visualized bands were quantified by using Quantity One software on a GS-800 densitometer (Bio-Rad Laboratories, Hercules, CA, USA). The antibodies used were as follows: CNN1/ calponin rabbit anti-human monoclonal (EP798Y) antibody (LifeSpan Biosciences, Inc., Seattle, WA, USA), Vimentin (D21H3) XP rabbit anti-human mAb (Cell Signaling Technology, Danvers, MA, USA), CTSB/cathepsin $B$ rabbit anti-human polyclonal antibody (LifeSpan Biosciences, Inc.), TAGLN/SM22 rabbit anti-human polyclonal (C-terminus) antibody (LifeSpan Biosciences, Inc.),
galectin-3/LGALS3 rabbit anti-human polyclonal antibody (Cell Signaling Technology), rabbit anti-mouse Akt polyclonal antibody (Cell Signaling Technology), Phospho-Akt (Ser473) (D9E) rabbit anti-human monoclonal antibody (Cell Signaling Technology), p44/42 MAPK (Erk1/2) (137 F5) rabbit anti-rat monoclonal antibody (Cell Signaling Technology), Phospho-p44/42 MAPK (Erk1/2) (Thr202/Tyr204) (D13.14.4E) rabbit anti-human monoclonal antibody (Cell Signaling Technology), and $\beta$-actin (13E5) rabbit anti-human monoclonal antibody (Cell Signaling Technology).

\section{RNA extraction and quantitative real-time polymerase chain reaction}

Total RNA was extracted by using a MicroElute Total RNA Kit (Omega Bio-Tek, Norcross, GA, USA). The comparative delta-delta threshold cycle $(\mathrm{Ct})$ method was adopted to analyze gene products by using the SYBR Select Master Mix (Applied Biosystems) in a 7500 Real-Time Polymerase Chain Reaction System (Applied Biosystems). Glyceraldehyde 3-phosphate dehydrogenase $(G A P D H)$ was used as an internal control gene to calculate the relative gene expression. The experiment was repeated three times, and the results were expressed as the mean \pm standard deviation. The primer sequences are reported in Additional file 1: Table S3.

\section{Short interfering RNA transfection}

Stealth short interfering RNA (siRNA) (Invitrogen, part of Life Technologies) against vimentin was transfected into the BM-MSCs. Non-targeting siRNA was used as a control. The experiment was conducted in accordance with the protocol of the manufacturer (Invitrogen). Briefly, the cells were harvested and subcultured in antibiotic-free medium for 12 hours. Then, the cells were transfected when the rate of cell fusion reached $50 \%$. The siRNAs were incubated with Lipofectamine 2000 (Invitrogen) for approximately 20 to 30 minutes and diluted with Opi-MEM medium (Gibco). The transfection mixture was gently added to the culture medium. Six hours later, the culture medium was replaced with fresh medium.

\section{Lentivirus-mediated overexpression in cell culture}

UC-MSCs were plated in $60-\mathrm{mm}$ dishes at a density of $5 \times 10^{5}$ cells per dish. After 24 hours of cultivation, the cells were infected with a sham control lentivirus or lentivirus encoding porcine vimentin (LV5-NC or LV5vimentin, respectively; GenePharma, Shanghai, China) at a multiplicity of infection of 100 in DMEM-F12 containing 10\% fetal bovine serum (FBS). Twelve hours later, these cells were processed for Western blotting and migration analysis. 


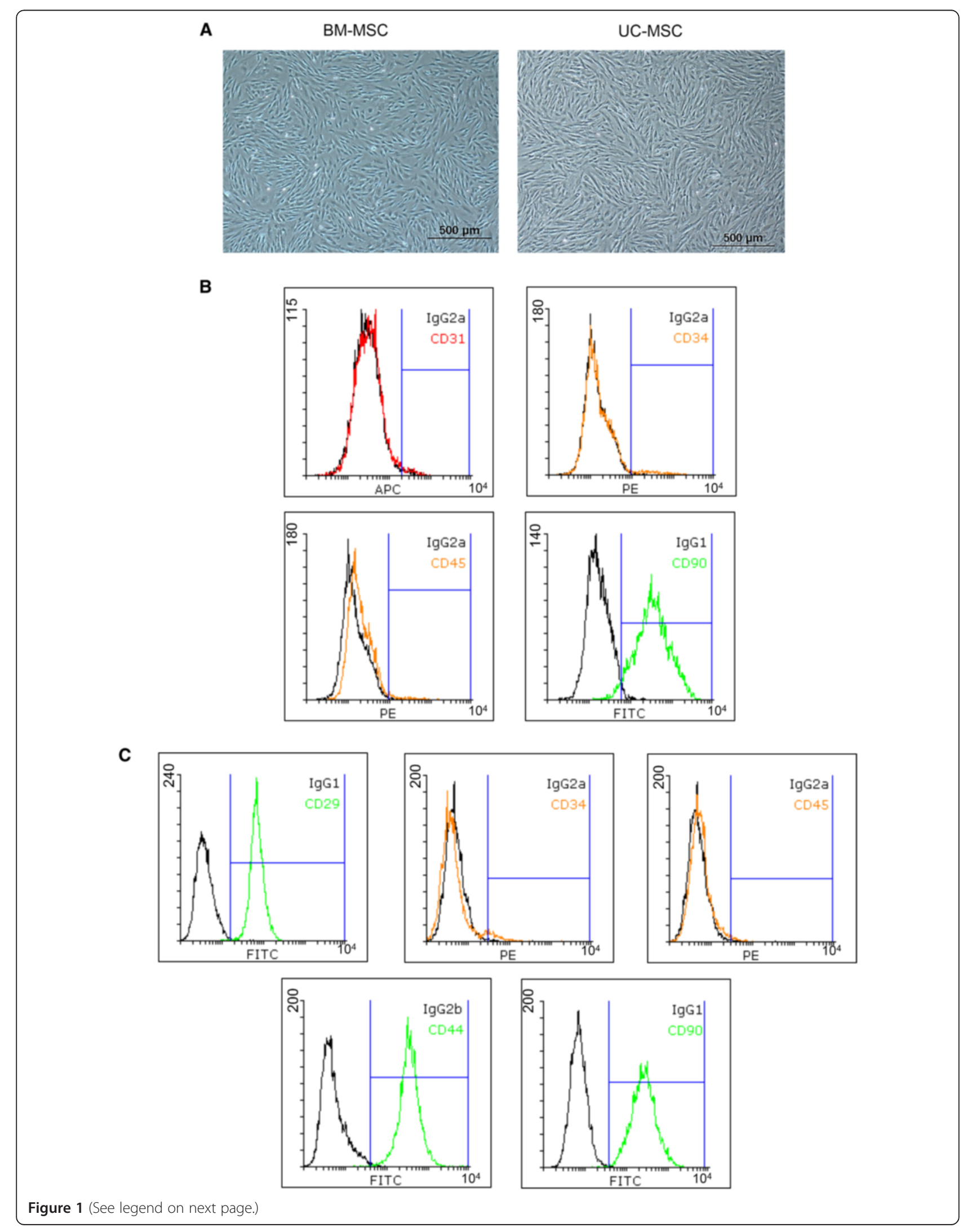


(See figure on previous page.)

Figure 1 Mesenchymal stem cells were spindle-like and expressed certain protein markers on the cell surface. (A) Cell shape and growth states were similar between bone marrow mesenchymal stem cells (BM-MSCs) and umbilical cord mesenchymal stem cells (UC-MSCS) in vitro. (B) As shown in the overlay histograms, five cell surface markers of BM-MSCs were verified: CD29 (FITC, green), CD44 (FITC, green), and CD90 (FITC, green), which were positive, and CD34 (PE, orange) and CD45 (PE, orange), which were negative. (C) Four cell surface markers of UC-MSCs were verified: CD90 (FITC, green), which was positive, and CD31 (APC, red), CD34 (PE, orange), and CD45 (PE, orange), which were negative. FITC, fluorescein isothiocyanate; PE, phycoerythrin.

\section{Mesenchymal stem cell migration analyzed by a scratch assay and by a transwell migration assay}

MSC migration was assessed by using a scratch assay and a transwell migration assay. The detailed process was as follows: Cells were cultured in $60-\mathrm{mm}$ dishes to confluence and incubated with $10 \mu \mathrm{g} / \mathrm{mL}$ mitomycin-C for 2 hours. The growth-arrested cells were transferred into 24-well plates at a density of $8 \times 10^{4}$ per well. Six hours later, 'scratches' were made along the bottom of the dish by using a $200-\mu \mathrm{L}$ pipette tip. Then, the cells were cultured for another 48 hours. During incubation, dishes were placed under a phase-contrast microscope to acquire images at selected time points $(6,12,24,36$, and 48 hours).

The cells were placed in the upper chamber of the transwell assembly $(6.5-\mathrm{mm}$ diameter inserts, $8.0-\mu \mathrm{m}$ pore size; Corning Costar, Corning, NY, USA) with $100 \mu \mathrm{L}$ of FBS-free medium at a density of $5 \times 10^{4}$ or $2 \times 10^{5}$ cells $/ \mathrm{mL}$ in $200 \mu \mathrm{L}$, and $800 \mu \mathrm{L}$ of medium containing $10 \%$ FBS as a source of chemoattractants filled the lower compartments. Cell number in the upper chamber of the transwell was not a coincidence among the three series of migration assays. For example, the cell number was $4 \times 10^{4}$ per well in the migration assay of wild-type BM-MSCs and UC-MSCs or Vimentinoverexpressed BM-MSCs, respectively. However, in the migration assay of Vimentin-knocked-down UC-MSCs, the cell number was only $1 \times 10^{4}$. After incubation at $37^{\circ} \mathrm{C}$ for 12 hours, the membrane was stained with Hoechst 33342 (Beyotime Institute of Biotechnology, Haimen, China), and the number of migrating cells was determined by counting 10 random fields per well under a fluorescence microscope at $40 \times$ magnification. Experiments were performed in sets of three for each group.

\section{Results}

Isolation, culture, and cell marker detection of porcine mesenchymal stem cells

The cell type and growth state of porcine BM-MSCs were similar to those of UC-MSCs (Figure 1A). The supernatant of porcine UC-MSCs was plated in cell culture dishes. The cells were generally spindle-like and of different sizes, and the nucleus could not be identified. After 48 hours of cultivation in vitro, a few cells climbed from the edges of adherent tissue. During cultivation, the cells displayed colony growth, and most of the cells were spindle-like, although there were several polygonallike cells as well. When the proliferating cell community integrated, the cells were arranged directionally, showing vortex-like growth (Figure 1A).

The purity of UC-MSCs and BM-MSCs was determined by flow cytometry. Cells with fluorescence in the range of M1 were considered positive cells, which were recognized by antibody detection. The auto-fluorescence intensity was less than $10^{1}$. The cells detected in this range were negative. Overlay histogram of each cell marker and its isotype antibody was made with Flowing Software (version 2.5.1). UC-MSC verification was performed by surface antigen expression, including the following four markers: positive for CD90, negative for vascular endothelial cell marker antigen CD31, negative for hematopoietic stem cell marker antigen CD34, and negative for leukocyte marker antigen CD45 (Figure 1B) (Additional file 1: Figure S3). The BM-MSC verification included the following five markers: positive for CD29, CD44, and CD90 and negative for CD34 and CD45 (Figure 1C) (Additional file 1: Figure S4).

\section{Porcine mesenchymal stem cells can differentiate into adipocytes and osteoblasts by in vitro induction}

Osteogenic MSCs were stained with Alizarin Red. Clumps or a sheet of orange-red precipitate appeared in the intercellular space and were regarded as calcium nodules on the cell surface (Figure 2A). Adipogenic MSCs were stained by Oil Red. Orange fat particle drops in cells were regarded as fat synthesized during differentiation (Figure 2B). These results indicate that porcine MSCs have the ability to differentiate in vitro. The osteogenic and adipogenic differentiation potentials were used as the evaluation criteria of MSCs in our work. We did not conduct any quantitative assessment of osteogenic, adipogenic, and chondrogenic differentiation.

\section{Analysis of differentially expressed proteins detected in iTraqs and gene ontology enrichment analyses of these proteins}

The samples were detected by using two independent iTRAQ-MS replicates. The DEPs were identified as proteins that had an absolute value of the log of the ratio of protein expression levels in UC-MSCs compared with BM-MSCs greater than 0.144 (specifically, the protein level between UC-MSCs and BM-MSCs differed by at 


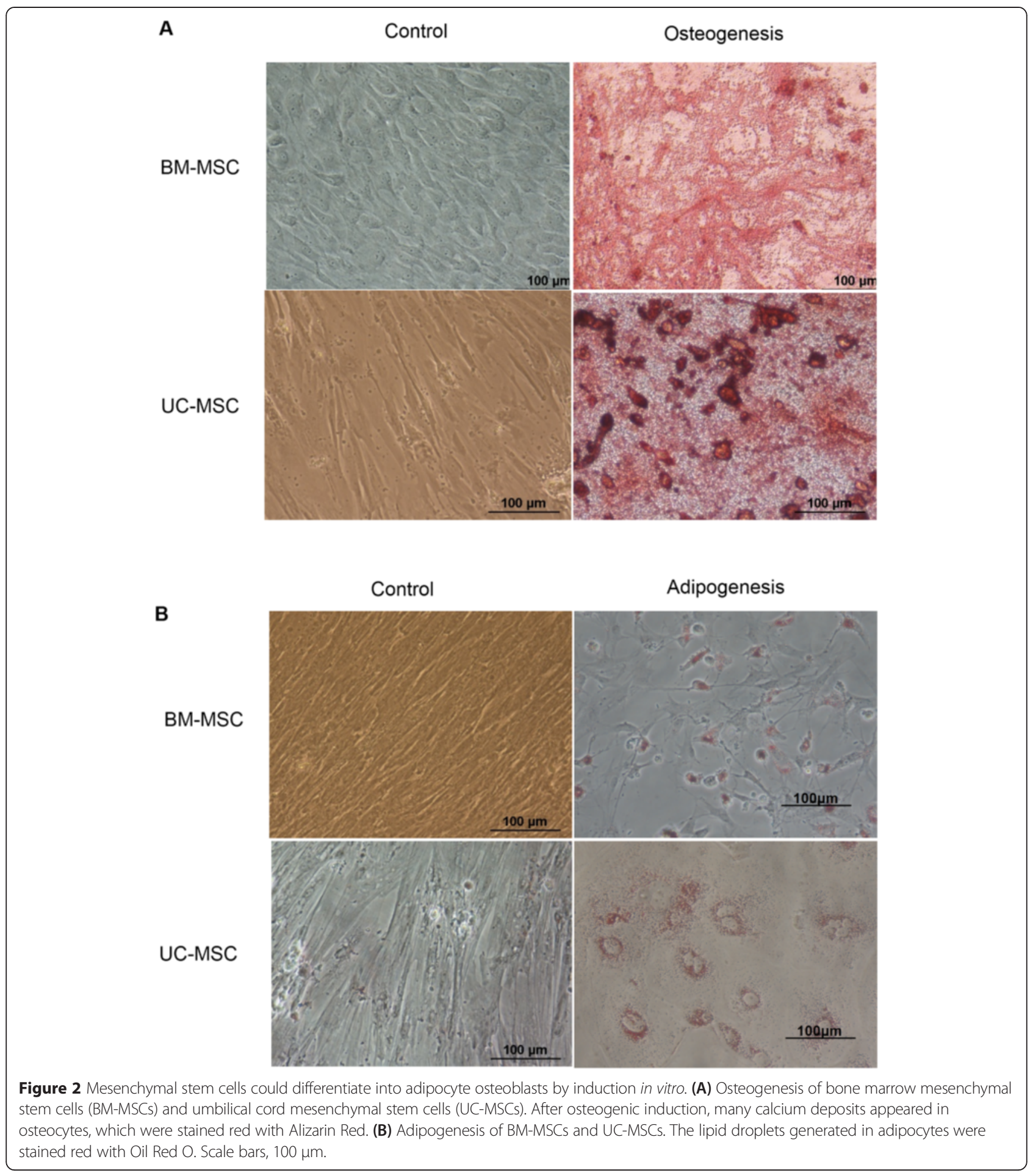

least 39\%). As a result, 83 and 79 DEPs were detected in the first and second MS replicates, respectively, and 95 DEPs were detected in total (Figure $3 \mathrm{~A}$ and Table 1). There were five proteins whose expression levels between UC-MSCs and BM-MSCs were at least 10-fold different, namely, LGALS3, VIM, TMSB4X, CALD1, and P4HA1 (Table 1). In total, 67 DEPs were detected in both MS replicates, and the differential trend of these proteins is consistent between the replicates (Figure 3A and Table 1). In addition, the reproducibility of the 
A

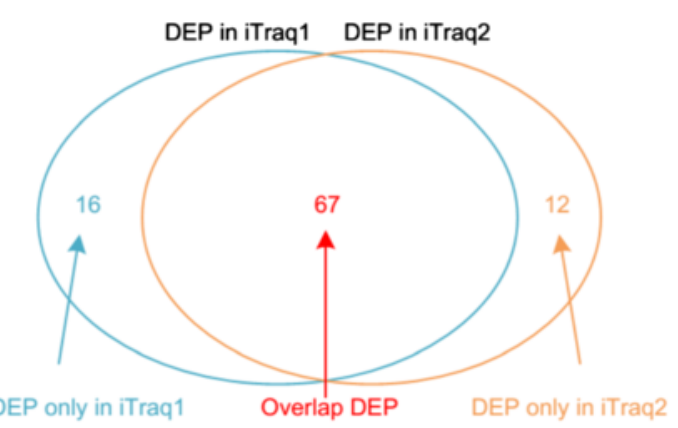

C

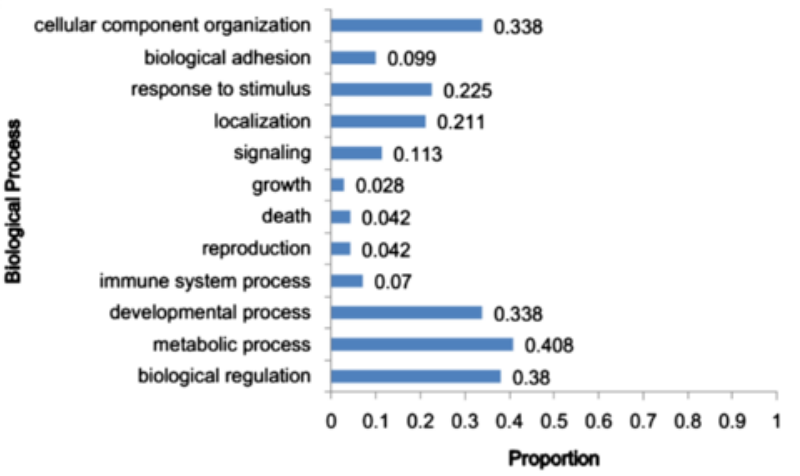

D extracellular vesicular exosome $]=0.099$

extracellular matrix $=0.07$

$$
\text { cell surface }=0.07
$$$$
\text { E. plasma membrane } 0.239
$$$$
\text { cytoskeleton } 0.282
$$$$
\text { vacuole }=0.042
$$

Golgi apparatus $=0.028$

E

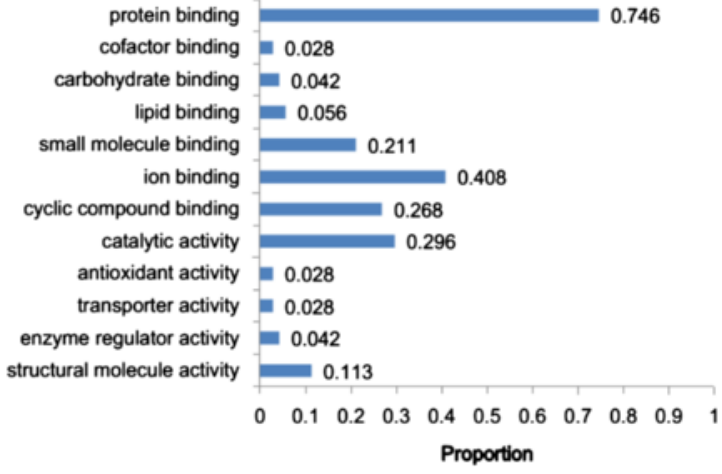

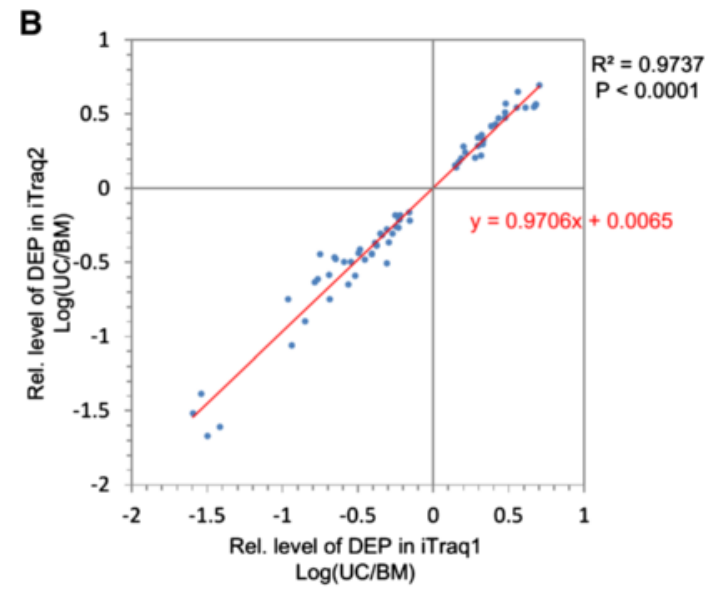


(See figure on previous page.)

Figure 3 Summary of differentially expressed proteins (DEPs) detected in two iTraq experiments and Gene Ontology enrichment analyses of DEPs. (A) Overview of DEPs in two iTraq experiments. (B) An x-y scatter plot used to analyze the consistency of two sets of iTraq data, whereby the x-axis represents the log value of the protein level in the UC-MSC-to-BM-MSC (UC/BM) ratio in the first mass spectrometry detection, and the $y$-axis represents that parameter in the second detection. Histograms showing DEP enrichment in biological processes (C), cellular components (D), and molecular functions (E). The enrichment degree was described by the ratio of the number of targeted proteins to all the annotated proteins. BM-MSC, bone marrow mesenchymal stem cell; iTraq, isobaric tagging for relative and absolute quantitation; UC-MSC, umbilical cord mesenchymal stem cell.

differential protein levels between the technical replicates was assessed by using a scatter plot (Figure 3B). Briefly, the $\mathrm{x}$-axis represents the log value of the iTRAQ ratios of the UC-MSCs to BM-MSCs in the first MS replicate, and the $y$-axis represents the iTRAQ ratios from the second replicate. This scatter plot can be fit linearly (Figure 3B) to obtain $\mathrm{R}^{2}$ and $P$ values of 0.9737 and less than 0.0001 , respectively. Of the 95 DEPs, 89 were annotated with GO terms by using the Sus scrofa GO annotations database and European Bioinformatics Institute Gene Ontology Annotation; we retrieved GO annotations of all of these proteins, and the annotations are listed in Additional file 1: Table S1. The GO enrichment analysis of biological processes, cellular components, and molecular functions showed that the enrichment degrees of DEPs in biological regulation, metabolic processes, developmental processes, immune system processes, reproduction, death, growth, signaling, localization, response to stimulus, biological adhesion, and cellular component organization are $0.38,0.408,0.338,0.07,0.042,0.042,0.028,0.113,0.211$, $0.225,0.099$, and 0.338 , respectively (Figure 3C). The degrees of DEP enrichment in the ribosome, nucleus, mitochondrion, endosome, endoplasmic reticulum, Golgi apparatus, vacuole, cytoskeleton, plasma membrane, cell surface, extracellular matrix, and extracellular vesicular exosome are 0.028, 0.254, 0.099, 0.028, 0.113, 0.028, 0.042, $0.282,0.239,0.07,0.07$, and 0.099, respectively (Figure 3D). The degrees of DEP enrichment in structural molecule activity, enzyme regulator activity, transporter activity, antioxidant activity, catalytic activity, cyclic compound binding, ion binding, small molecule binding, lipid binding, carbohydrate binding, cofactor binding, and protein binding are 0.113, 0.042, 0.028, 0.028, 0.296, $0.268,0.408,0.211,0.056,0.042,0.028$, and 0.746 , respectively (Figure 3E).

KEGG pathway analysis of differentially expressed protein indicated a remarkable difference in the motility capacity between UC-MSCs and BM-MSCs

In total, 20 of 95 DEPs were mapped by using the KEGG pathway database, and 37 pathways were obtained (Additional file 1: Table S2). The primary pathways of the DEPs include energy metabolism, amino acid metabolism, translation, folding-sorting degradation, signal transduction, cell motility, cell communication, and immune system (Figure 4A). Weighing the fold changes of proteins in each pathway determined that the weight in the cell motility pathway is the highest (Figure 4A). Moreover, 11 of 20 proteins could participate in this pathway (Figure $4 \mathrm{~B}$ ) via mapping and linking-related pathways. The results strongly suggest that cell motility, including migration, invasion, and adhesion, may be markedly different between UC-MSCs and BM-MSCs.

\section{Conformation of the differential expression of five proteins}

Significantly DEPs are most likely to affect cellular biological behavior. To verify the quantitative proteomic results, we chose Vimentin and LGALS3 because they exhibited large differences in expression between the two types of MSCs, whereas CTSB, TAGLN, and CNN1 were randomly selected for Western blotting verification (Figure 5). The Western blot results are in agreement with the iTRAQ results. Therefore, the results of the Western blotting analysis confirmed the reliability of the proteomic analysis.

\section{The cell migration ability of BM-MSCs is higher than that of UC-MSCs}

The proteomic data displayed many DEPs related to cell migration, such as Vimentin, LGALS3, FSCN1, TAGLN, and CTSB (Table 1). Scratch and transwell assays were used to detect the migration ability of MSCs. The results of the migration assay showed that BM-MSCs had a greater migration ability than UC-MSCs (Figure 6A, B).

\section{Vimentin protein positively modulates mesenchymal stem} cell migration

Additionally, we found that the expression of the vimentin gene is higher in BM-MSCs than in UC-MSCs (Table 1). The Vimentin protein has been positively related to the high metastasis of tumor cells and lymphocytes. Therefore, vimentin may play an important role in MSC migration. To test this function, we knocked down vimentin in BM-MSCs and overexpressed this 
Table 1 Differentially expressed proteins detected by two iTraq experiments

\begin{tabular}{|c|c|c|c|c|}
\hline $\begin{array}{l}\text { UniProt accession } \\
\text { number }\end{array}$ & Protein name & Gene name & $\begin{array}{l}\text { Level in iTraq1 } \\
\log (\mathrm{UC} / \mathrm{BM})\end{array}$ & $\begin{array}{l}\text { Level in iTraq2 } \\
\log (\mathrm{UC} / \mathrm{BM})\end{array}$ \\
\hline A3EX84 & Galectin & LGALS3 & -1.50000007 & -1.66800008 \\
\hline P02543 & Vimentin & VIM & -1.59599994 & -1.51599999 \\
\hline B3XXC3 & N/A & TMSB4X & -1.41600004 & -1.60800006 \\
\hline F1SNH3 & N/A & CALD1 & -1.54000006 & -1.38400002 \\
\hline A1X898 & N/A & P4HA1 & -0.93999986 & -1.05999998 \\
\hline F1SU97 & N/A & PSAP & -0.94400011 & \\
\hline F1SKI0 & N/A & MYH11 & -0.85200015 & -0.89600002 \\
\hline F1SK03 & N/A & ANPEP & -0.96399985 & -0.7479999 \\
\hline Q56VQ1 & N/A & Oas2 & 0.827999957 & \\
\hline F1SEQ7 & N/A & FAM213A & & 0.772000048 \\
\hline F2Z5M2 & N/A & LOC100515138 & -0.68800004 & -0.7479999 \\
\hline F1RWW4 & N/A & PDLIM5 & -0.78800002 & -0.632 \\
\hline Q2YGT9 & 605 ribosomal protein L6 & RPL6 & 0.70400004 & 0.695999967 \\
\hline D0G7F7 & N/A & TPM4 & -0.76800012 & -0.61199992 \\
\hline F1RQW2 & N/A & C4 & & -0.68800004 \\
\hline A9YUA9 & N/A & $N / A$ & -0.692 & -0.58400011 \\
\hline A9GYW6 & N/A & APLE & -0.63599995 & \\
\hline B2LUG8 & N/A & $N / A$ & 0.680000019 & 0.568000024 \\
\hline F1RKW9 & N/A & MYO1D & 0.676000031 & 0.5599999932 \\
\hline F1SDX6 & N/A & TGM2 & 0.667999999 & 0.548000035 \\
\hline F1S663 & N/A & LAMC1 & 0.5599999932 & 0.652000004 \\
\hline F1SKJ1 & N/A & MYH9 & -0.56399995 & -0.64799991 \\
\hline Q9TSX9 & Peroxiredoxin-6 & PRDX6 & -0.752 & -0.44400006 \\
\hline F1RGS2 & N/A & GBA & 0.612000045 & 0.544000055 \\
\hline F1SLI3 & Microtubule-associated protein & MAP4 & -0.64799991 & -0.47600007 \\
\hline F1SMN1 & N/A & CALU & -0.65599994 & -0.46399992 \\
\hline F2Z557 & N/A & $P A B P C 1$ & -0.51999998 & -0.58800002 \\
\hline C6K7I0 & Importin subunit alpha & N/A & 0.551999967 & 0.544000055 \\
\hline P20112 & SPARC & SPARC & -0.59199998 & -0.496 \\
\hline P27594 & Interferon-induced GTP-binding protein Mx1 & MX1 & 0.480000049 & 0.572000047 \\
\hline F1S4Y8 & N/A & LOC100621044 & & -0.52399996 \\
\hline F1RSC3 & N/A & SCPEP1 & & 0.52399994 \\
\hline COLZLO & Fascin & FSCN1 & -0.548 & -0.496 \\
\hline F1RJL6 & N/A & CLIP2 & -0.51199996 & \\
\hline F1SAP4 & N/A & WARS & & -0.5080001 \\
\hline Q08092 & Calponin-1 & CNN1 & & 0.508000041 \\
\hline F1RST0 & N/A & $\mathrm{HSPH1}$ & 0.476000051 & 0.512000015 \\
\hline F2Z5K2 & Proteasome subunit alpha type & PSMA5 & 0.476000051 & 0.476000051 \\
\hline F1RRV6 & N/A & NDRG1 & -0.45599997 & -0.48000003 \\
\hline F1SJS8 & N/A & TAGLN & -0.496 & -0.43599995 \\
\hline F1SLA0 & ATP synthase subunit beta & ATP5B & 0.431999947 & 0.47199996 \\
\hline F1SQ11 & N/A & EEA1 & -0.48799998 & -0.41200004 \\
\hline
\end{tabular}


Table 1 Differentially expressed proteins detected by two iTraq experiments (Continued)

\begin{tabular}{|c|c|c|c|c|}
\hline F1S764 & N/A & CPT2 & & 0.427999945 \\
\hline A5A768 & N/A & AP3D1 & 0.4279999945 & \\
\hline F1S554 & N/A & PALMD & -0.40800001 & -0.44400006 \\
\hline F1SGP8 & N/A & RCN1 & -0.40800001 & -0.43999996 \\
\hline F1SMN5 & N/A & FLNC & 0.41199995 & 0.431999947 \\
\hline F1S827 & N/A & SERBP1 & -0.41599992 & \\
\hline F1SMV6 & N/A & LOC100737174 & -0.30800003 & -0.50399997 \\
\hline A8CYB8 & N/A & $R / G-I$ & 0.384000008 & 0.420000017 \\
\hline F1SIJ9 & N/A & PSAT1 & -0.39999997 & \\
\hline F1SV06 & N/A & PABPC4 & -0.37600003 & -0.38400001 \\
\hline F1RKG8 & N/A & PEBP1 & -0.37600003 & -0.38400001 \\
\hline F1S9A4 & N/A & NUCB2 & -0.38799997 & -0.36800001 \\
\hline $\mathrm{F} 2 \mathrm{Z} 5 \mathrm{C} 1$ & Annexin & ANXA5 & 0.360000074 & \\
\hline B6CVD6 & N/A & TXNDC4 & 0.344000043 & \\
\hline F1SC51 & N/A & $N / A$ & & 0.344000043 \\
\hline F1RI39 & N/A & LOC100517284 & 0.3199999994 & 0.364000018 \\
\hline F1SPP8 & N/A & CKAP4 & -0.29599998 & -0.36400003 \\
\hline F1SJJ5 & N/A & $R P L 4$ & 0.331999906 & 0.327999994 \\
\hline F1RJ93 & N/A & TAGLN2 & -0.33999999 & -0.31599999 \\
\hline F1SS24 & N/A & FN1 & -0.35199996 & -0.30400001 \\
\hline F1RRU7 & N/A & $M R C 2$ & -0.34399997 & -0.31199999 \\
\hline F1SGJ6 & N/A & SLMAP & 0.29600007 & 0.344000043 \\
\hline P33198 & Isocitrate dehydrogenase [NADP], mitochondrial & $\mathrm{IDH} 2$ & 0.327999994 & 0.299999937 \\
\hline F1RWJ5 & N/A & KPNB1 & 0.292000077 & \\
\hline F1S3M9 & N/A & EPB41L2 & 0.29600007 & 0.288000032 \\
\hline F1SSA6 & N/A & LOC396903 & -0.30800003 & -0.27600005 \\
\hline BOLY42 & N/A & N/A & -0.27199999 & -0.30400001 \\
\hline P28491 & Calreticulin & CALR & -0.28400001 & \\
\hline Q4GWZ2 & $40 S$ ribosomal protein SA & RPSA & 0.315999943 & 0.224000024 \\
\hline B2CNZ7 & N/A & CTSB & & -0.26399998 \\
\hline F1SIX3 & N/A & UGP2 & -0.24799998 & -0.25600002 \\
\hline F1SJR7 & N/A & TTLL12 & -0.23199998 & -0.26399998 \\
\hline G9F6X8 & N/A & $N / A$ & 0.275999909 & 0.208000125 \\
\hline F1RWT2 & N/A & PLS3 & 0.199999944 & 0.284000063 \\
\hline B5LOY2 & N/A & CAST & -0.24000005 & \\
\hline F1RGP1 & N/A & MYBBP1A & 0.211999977 & 0.244000118 \\
\hline Q5MJE5 & N/A & $N / A$ & & -0.22800001 \\
\hline Q06AT0 & Hippocalcin-like protein 1 & HPCAL1 & & -0.22800001 \\
\hline F1RI15 & N/A & HSPA4 & 0.220000031 & \\
\hline F1SFZ8 & N/A & TLN1 & -0.22800001 & -0.21199999 \\
\hline Q8SPT0 & N/A & N/A & & 0.21600007 \\
\hline DOGOC6 & Asparagine synthetase & ASNS & -0.224 & -0.20799999 \\
\hline F1RS36 & N/A & HSPA5 & -0.25200001 & -0.17999995 \\
\hline F1SQK1 & N/A & $N / A$ & -0.21999997 & -0.17999995 \\
\hline
\end{tabular}


Table 1 Differentially expressed proteins detected by two iTraq experiments (Continued)

\begin{tabular}{|c|c|c|c|c|}
\hline F1SOV3 & Annexin & ANXA6 & 0.183999971 & 0.203999991 \\
\hline F1SK12 & N/A & MAP1B & -0.15599999 & -0.21600001 \\
\hline F1SL58 & $\mathrm{N} / \mathrm{A}$ & LOC100519091 & -0.18400006 & \\
\hline Q59IP2 & N/A & COL5A2 & -0.18400006 & \\
\hline Q29092 & Endoplasmin & HSP90B1 & 0.167999844 & 0.179999932 \\
\hline F1S596 & $\mathrm{N} / \mathrm{A}$ & PRKCSH & -0.15999999 & -0.15999999 \\
\hline P80021 & ATP synthase subunit alpha, mitochondrial & ATP5A1 & 0.144000063 & 0.156000041 \\
\hline F1RR78 & $\mathrm{N} / \mathrm{A}$ & LOC100049693 & 0.151999844 & 0.144000063 \\
\hline BOLXK8 & N/A & HPRT1 & -0.144 & \\
\hline
\end{tabular}

iTraq, isobaric tagging for relative and absolute quantitation; N/A, not applicable; UC/BM, umbilical cord mesenchymal stem cell/bone marrow mesenchymal stem cell.

gene in UC-MSCs to assess its effect on cell migration (Additional file 1: Figure S1). As shown in Figure 6C and $\mathrm{D}$, the knockdown of vimentin in BM-MSCs limits their migration ability; however, vimentin overexpression in UC-MSCs promotes migration. These results suggested that the higher migration capability of BMMSCs may be due to the higher expression of migrationrelated protein than UC-MSCs.

\section{Discussion}

In recent years, adult MSCs have been widely used in regenerative medicine research, and these cells can be isolated from various tissues. Two types of MSCs were used in this study: UC-MSCs and BM-MSCs. These cells have similar biological characteristics and immunophenotypes. Because UC-MSCs have the advantages of both convenience and ease of cell isolation, they may be used as an alternative therapy candidate over BM-MSCs, which are more difficult to isolate [2]. In addition, comparative proteomic studies indicate that UC-MSCs have a high degree of overlap in protein expression profiles with MSCs compared with embryonic stem cells [4]. UC-MSCs also display differences in their osteogenic differentiation phenotypes. For example, UC-MSCs show few Alizarin Red-positive deposits compared with BMMSCs; however, the associated mechanism is unclear [17]. Interestingly, comparative proteomic analyses of porcine BM-MSCs and UC-MSCs have not been reported. Therefore, analyzing the DEPs between these two sets of porcine MSCs may reveal molecular mechanisms underlying the self-renewal, differentiation, and tissue homing capacity of these cells and may provide a theoretical basis for the clinical application of MSCs in cell therapy and regenerative medicine [1].

During cell therapy, the integration of the donor cells or biological material and the damaged tissue is dependent on cell migration, differentiation, and matrix remodeling $[2,17,18]$. In this study, BM-MSCs and UC-MSCs were isolated from newborn piglets, and their cell surface marker molecules, such as CD29, CD31, CD34, CD44, CD45, and CD90, were identified during osteogenic and adipogenic differentiation assays to determine the in vitro cell homozygosity of MSCs and to maintain pluripotency.

In this study, we used iTRAQ labeling coupled with LC-MS/MS to conduct a quantitative analysis of DEPs from porcine BM-MSCs and UC-MSCs. This method is not only gel-free but also sensitive and can detect many low-abundance proteins often missed by other proteomic methods. The results showed that 95 DEPs have at least a $39 \%$ difference in expression levels in two technical replicates. The DEPs include five proteins that are expressed 10 times more highly in BM-MSCs: LGALS3, VIM, TMSB4X, CALD1, and P4HA1. Three of these proteins were reviewed porcine proteins in the Swiss-Prot protein database, LGALS3, VIM, and TMSB4X, and two were automatically annotated, CALD1 and P4HA1. These proteins may play important roles in the process of cell biology.

We subjected 20 of the DEPs to KEGG pathway analysis and identified several pathways involved in many aspects of cell biology, including metabolism, genetic information processing, environmental information processing, cellular processes, and organismal systems. The most highly represented pathway for the DEPs is the cell motility pathway. Protein detection methods used in our experiment could analyze only the expression level of each protein between different MSCs. But variations in the level of phosphorylation of proteins would not be detected. And in different types of cells, effects of protein phosphorylation on pathway could hardly be compared. However, we attempted to detect the phosphorylation of AKt and Erk in MSCs which showed that phosphorylation of both proteins increased significantly in the BM-MSCs (Additional file 1: Figure S2).

Moreover, we observed that some structural proteins, which are important for structural support of the cell 


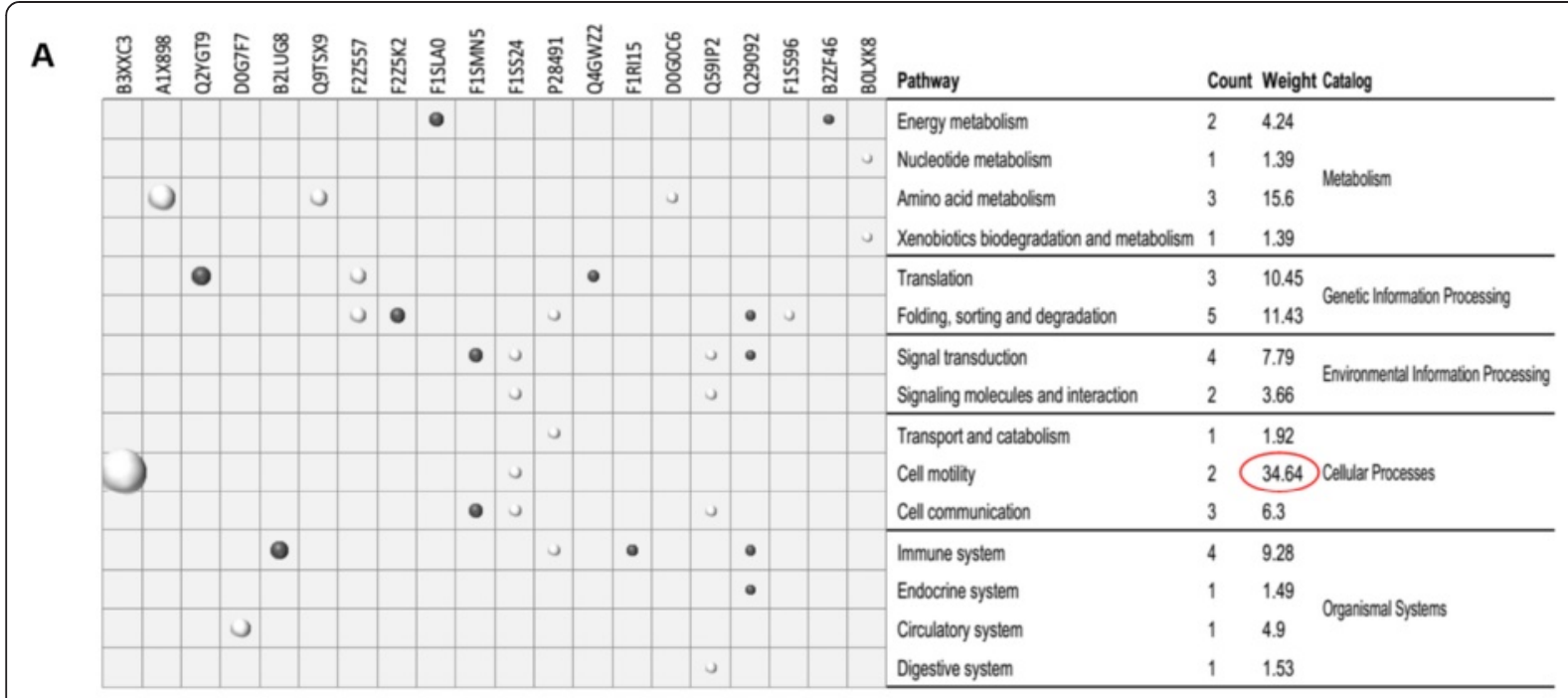

B

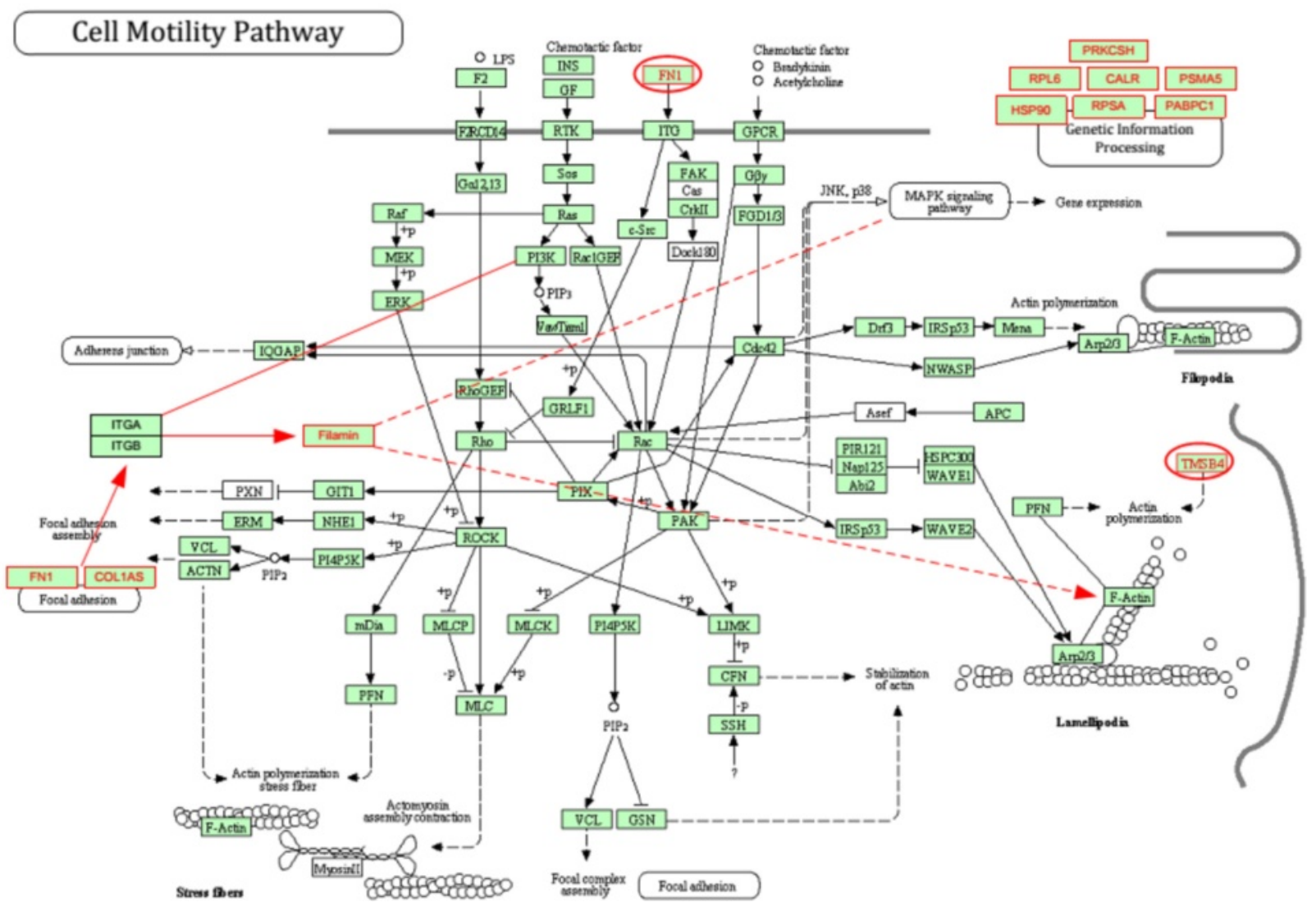

Figure 4 KEGG pathway analysis of DEPs indicated a remarkable difference in motility capacity between UC-MSCs and BM-MSCs. (A) A bubble graph describing the distribution of DEPs in a classified KEGG pathway. The bubble size represents the differential protein expression level. The dark bubbles show that the protein level in UC-MSCs is higher than that in BM-MSCs, whereas the light bubbles show the opposite trend. (B) A KEGG pathway map with additional manual annotations and links. The red boxes represent the DEPs detected in the experiment. The red boxes with red oval frames existed in the original KEGG pathway, and the red boxes with no oval frames and red links were added in line with a related KEGG pathway. BM-MSC, bone marrow mesenchymal stem cell; DEP, differentially expressed protein; KEGG, Kyoto Encyclopedia of Genes and Genomes; UC-MSC, umbilical cord mesenchymal stem cell. 


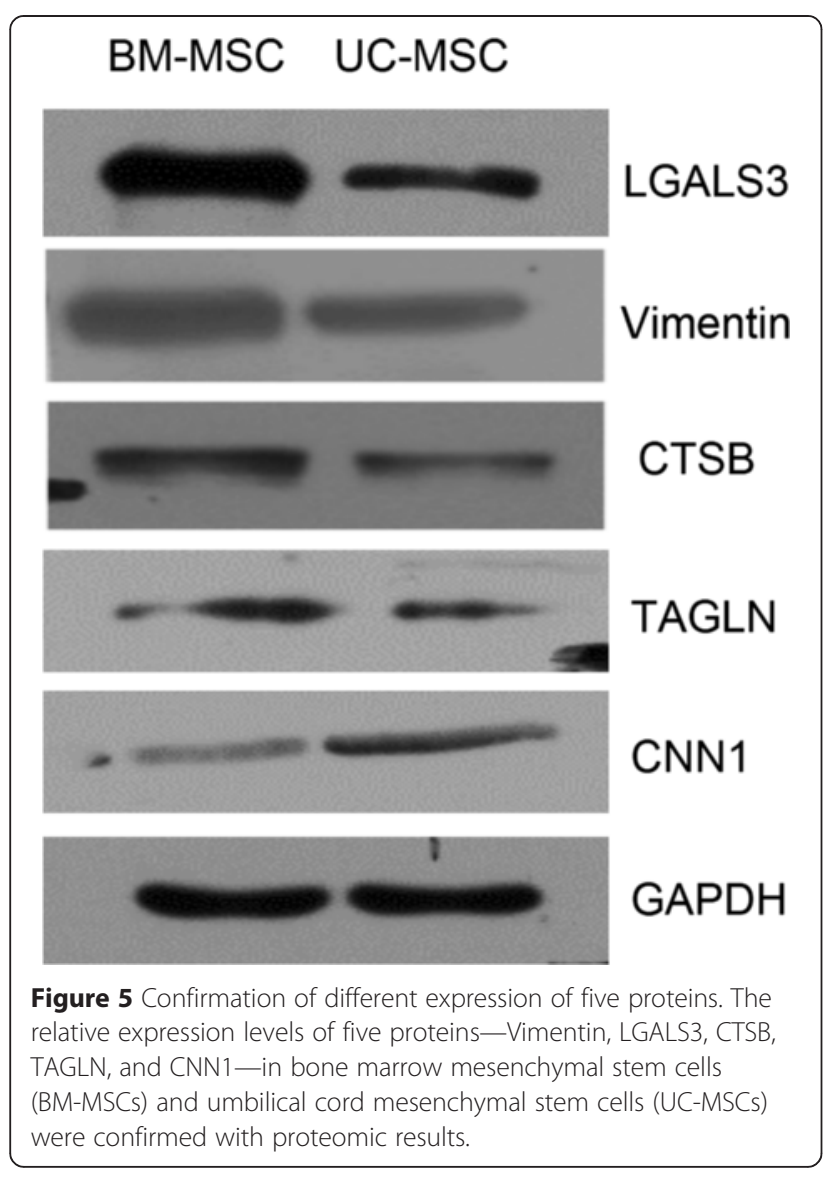

and maintaining the shape of the cell, were differentially expressed in these MSCs. Compared with UC-MSCs, VIM is upregulated, whereas CNN1, MYO1D, and PLS3 are downregulated in BM-MSCs. It has been shown that the expression of CNN1 in smooth muscle increased after transforming growth factor-beta (TGF $\beta$ ) induced BM-MSC differentiation [19]. In this study, the expression of CNN1 was downregulated in a time-dependent manner (data not published) during the osteogenic differentiation of BM-MSCs. Interestingly, the proteomic results showed that the CNN1 expression level in BMMSCs was half that of UC-MSCs. Moreover, the osteogenic differentiation experiment suggested that the differentiation ability of BM-MSCs is greater than that of UC-MSCs. Taken together, these results suggest that $\mathrm{CNN} 1$ may play an inhibitory role in the osteogenic differentiation of MSCs. These results suggest that the BMMSCs and UC-MSCs may have some differences in cell motility, including migration, invasion, and adhesion.

In these experiments, the pathway analysis was performed by weighting the analysis with protein abundance data to achieve a better understanding of the differences present in these MSCs. This methodology, compared with analysis methods that consider only the amount of proteins, more comprehensively highlight the different pathways activated in these cells.

Related reports regarding the proteomic differences between human BM-MSCs and UC-MSCs have been published. These studies showed that the expression of proteins that are positive regulators of cell migration, such as LGALS3, VIM, and TMSB4X, are significantly higher in BM-MSCs compared with UC-MSCs and that the expression levels of negative regulators are significantly reduced. Meanwhile, cell migration assays revealed a higher migration ability of BM-MSCs. The relationship between VIM and MSC migration is not well understood. Therefore, we compared the role of VIM in the migration phenotype of porcine BM-MSCs and UC-MSCs. Vimentin was identified in these experiments to be positively related in MSC migration. This type III intermediate filament protein is abundantly expressed in MSCs and in endothelial cells [20]. Although Vimentin is a basic regulatory protein involved in many physiological processes, such as intracellular homeostasis, cell viability, endothelial integrity, and nervous system injury, vimentin gene knockout mice showed no obvious barriers to embryonic development and had no effect on the survival of individuals [20,21]. To explore the role of the Vimentin protein in MSC migration, the expression of this protein was knocked down in BM-MSCs and overexpressed in UC-MSCs in our study. Scratch injury and transwell migration tests showed that the migration ability of BM-MSCs was significantly higher than that of UC-MSCs, and this ability is associated with the up-regulated expression of the Vimentin protein. In addition, Rogel MR et al. reported that, in alveolar epithelial cells, TGF $\beta 1$ could enhance the expression of Vimentin [22]. However, in our proteomic data, we did not find any difference in expression of TGF $\beta 1$ between BM-MSCs and UC-MSCs. When the vimentin gene was deleted, the expression of adhesion molecules was impaired, thereby reducing the adhesion and trans-endothelial migration rate of lymphocyte cells [23]. A close relation between Vimentin and cancer cell migration capabilities has also been found [24-26]. LGALS3, which is the third member of the galactosidase lectin protein family, was significantly increased in BMMSCs, and this protein plays an important role in cell proliferation, adhesion, migration, and apoptosis [27-32].

The expression of TMSB4X, a major G-actin-sequestering peptide that is largely distributed in various cells and in the circulatory system and that is capable of binding to G-actin to inhibit actin aggregation, was significantly more highly expressed in BM-MSCs than in UC-MSCs [33,34]. TMSB4X promoted increased expression of MMP-1, MMP-2, and MMP-3 as well as the secretion of plasminogen activator inhibitor-1 (PAI-1), 


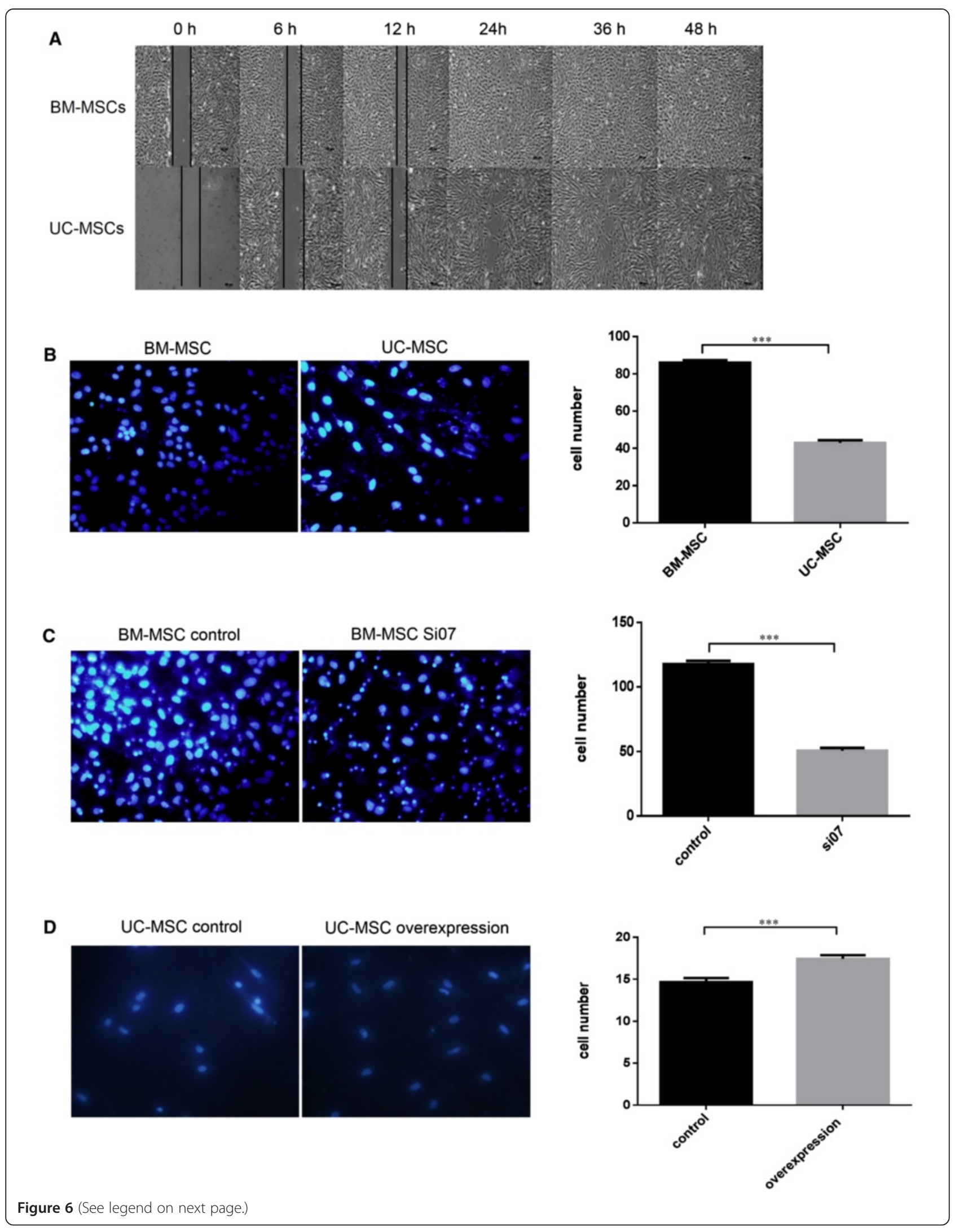


(See figure on previous page.)

Figure 6 The cell migration ability of bone marrow mesenchymal stem cells (BM-MSCs) was higher than that of umbilical cord mesenchymal stem cells (UC-MSCs), which might be related to Vimentin. (A) Zero, six, 12, 24, 36, and 48 hours after scratching in BM-MSCs and UC-MSCs, respectively. Downward lines were drawn to show the healing rate of each scratching even. (B) Transwell results of wild-type BM-MSCS and UC-MSCS. (C, D) The effect of Vimentin expression on the migration of BM-MSCs and UC-MSCs. The expression of Vimentin was reduced in BM-MSCS (C) and elevated in UC-MSCs (D). As the histograms showed, the migration effect of BM-MSCs is higher than that of UC-MSCs and positively correlated with the expression of Vimentin. ${ }^{* * *} P<0.01$. Bars show the standard error of the mean. Magnifications: $4 \times(A)$ and $40 \times(B-D)$.

thereby increasing the levels of cell adhesion and migration [35]. The regulation of cell migration by TMSB4X is cell-specific. In endothelial cells, the transcription level of PAI-1 is enhanced by TMSB4X [35]. However, comparative proteomic studies of BM-MSCs and UCMSCs showed that the expression of PAI-1 in BM-MSCs was significantly lower than that in UC-MSCs, and the cell migration assay results indicated that PAI-1 is a negative regulator of cell migration in MSCs [2]. Thus, the effect of TMSB4X on endothelial cell migration is most likely regulated by a complex variety of factors. In tumor cells, TMSB4X can promote cell migration and vascular endothelial growth factor-induced angiogenesis, thereby accelerating tumor growth and metastasis $[36,37]$. Lastly, TMSB4X is an anti-inflammatory factor that could accelerate the repair of skin and corneal burns, a process which is closely related to cell migration promoted by TMSB4X [38].

One interesting result of this study is that proteins related to the immune response, such as PRKCSH, Oas2, and RIG-I, are differentially expressed between BMMSCs and UC-MSCs. PRKCSH is a regulatory subunit of glucosidase II and is expressed predominantly in the endoplasmic reticulum [39]. In fetal tissue, PRKCSH is expressed in the ductal plate, bile ducts, and hepatocytes, suggesting that PRKCSH is most likely required for bile duct development [39]. RIG-I is a member of the RIG-I-like receptor family and plays a major role in pathogen sensing of RNA virus infection to induce type I interferon production [40]. However, strong evidence has shown that significant induction of RIG-I occurs during normal myelopoiesis and that the development of a progressive myeloproliferative disorder is disrupted when Rig-I is knocked down [41].

\section{Conclusions}

This study is the first of its kind to perform a quantitative analysis of DEPs between porcine BM-MSCs and UCMSCs, combined with GO analysis, KEGG signaling pathway analysis, and cell migration studies. These experiments describe differences in the migration abilities of these two types of cells. The data indicate that this difference in migration is related to the expression levels of proteins, and therefore, they provide experimental evidence for cell-based targeted therapy using MSCs.

\section{Additional file}

Additional file 1: Table S1. Gene Ontology annotations of differentially expressed proteins. Table S2. Kyoto Encyclopedia of Genes and Genomes (KEGG) pathways of differentially expressed proteins. Table S3. Primer information. Figure $\mathbf{S 1}$. Results of real-time polymerase chain reaction (PCR) and Western blotting of mesenchymal stem cells (MSCs). Figure S2. Phosphorylation level detection of AKt and Erk in mesenchymal stem cells (MSCs). Figure S3. Histograms of immunophenotypic profiling of bone marrow mesenchymal stem cells (BM-MSCs). Figure S4. Histograms of immunophenotypic profiling of umbilical cord mesenchymal stem cells (UC-MSCS).

\section{Abbreviations}

BM-MSC: bone marrow mesenchymal stem cell; DEP: differentially expressed protein; DMEM: Dulbecco's modified Eagle's medium; FBS: fetal bovine serum; GO: Gene Ontology; HPLC: high-performance liquid chromatography; iTRAQ: isobaric tag for relative and absolute quantitation; KEGG: Kyoto Encyclopedia of Genes and Genomes; LC-MS/MS: liquid chromatographytandem mass spectrometry; MS: mass spectrometry; MSC: mesenchymal stem cell; MS/MS: tandem mass spectrometry; PAI-1: plasminogen activator inhibitor-1; PBS: phosphate-buffered saline; PSI: pounds per square inch; SCX: strong cation exchange; siRNA: short interfering RNA; TGFß1: transforming growth factor-beta; TOF: time-of-flight; UC-MSC: umbilical cord mesenchymal stem cell.

\section{Competing interests}

The authors declare that they have no competing interests.

\section{Authors' contributions}

$\mathrm{LH}$ performed most of the experiments and produced most of the figures. CN, BW, SY, and SF analyzed the data. WZ and WH performed experiments of cell migration. $L L$ and TW isolated pig MSCs. $K L, L Z$, and $Y M$ supervised the project, designed experiments, and wrote the manuscript. All authors read and approved the final manuscript.

\section{Acknowledgments}

This project was supported by the National Natural Science Foundation of China (31272404, 31330074, and 81370235), the National High Technology Research and Development Program of China (2012AA020603), the '973' National S\&T Major Project (2010CB912504 and 2011CB503900), and the Agricultural Science and Technology Innovation Program (ASTIP-IAS05) of the Chinese Academy of Agricultural Sciences.

\section{Author details}

${ }^{1}$ State Key Laboratory of Animal Nutrition and Key Laboratory of Farm Animal Genetic Resources and Germplasm Innovation of Ministry of Agriculture, Institute of Animal Sciences, Chinese Academy of Agricultural Sciences, No. 2 Yuanmingyuan West Road, Haidian District 100193, Beijing, China. ${ }^{2}$ The Institute of Cardiovascular Sciences and Institute of Systems Biomedicine, School of Basic Medical Sciences, and Key Laboratory of Molecular Cardiovascular Sciences of Ministry of Education, Peking University Health Science Center, No. 38 Xueyuan Road, Haidian District 100191, Beijing, 
China. ${ }^{3}$ Cleveland Clinic Lerner Research Institute Mass Spectrometry Laboratory for Protein Sequencing, Euclid Avenue, Cleveland, OH 44195, USA.

Received: 5 September 2014 Revised: 14 September 2014 Accepted: 24 March 2015

Published online: 15 April 2015

\section{References}

1. Kim HS, Choi DY, Yun SJ, Choi SM, Kang JW, Jung JW, et al. Proteomic analysis of microvesicles derived from human mesenchymal stem cells. J Proteome Res. 2012;11:839-49.

2. Li G, Zhang XA, Wang H, Wang X, Meng CL, Chan CY, et al. Comparative proteomic analysis of mesenchymal stem cells derived from human bone marrow, umbilical cord, and placenta: implication in the migration. Proteomics. 2009;9:20-30.

3. Izadpanah R, Trygg C, Patel B, Kriedt C, Dufour J, Gimble JM, et al. Biologic properties of mesenchymal stem cells derived from bone marrow and adipose tissue. J Cell Biochem. 2006;99:1285-97.

4. Roche S, Delorme B, Oostendorp RA, Barbet R, Caton D, Noel D, et al. Comparative proteomic analysis of human mesenchymal and embryonic stem cells: towards the definition of a mesenchymal stem cell proteomic signature. Proteomics. 2009;9:223-32.

5. Li M, Wu H, Luo Z, Xia Y, Guan J, Wang T, et al. An atlas of DNA methylomes in porcine adipose and muscle tissues. Nat Commun. 2012;3:850.

6. Wagner W, Wein F, Seckinger A, Frankhauser M, Wirkner U, Krause U, et al. Comparative characteristics of mesenchymal stem cells from human bone marrow, adipose tissue, and umbilical cord blood. Exp Hematol. 2005;33:1402-16.

7. Mrozik KM, Zilm PS, Bagley CJ, Hack S, Hoffmann P, Gronthos S, et al. Proteomic characterization of mesenchymal stem cell-like populations derived from ovine periodontal ligament, dental pulp, and bone marrow: analysis of differentially expressed proteins. Stem Cells Dev. 2010;19:1485-99.

8. Kim J, Shin JM, Jeon YJ, Chung HM, Chae Jl. Proteomic validation of multifunctional molecules in mesenchymal stem cells derived from human bone marrow, umbilical cord blood and peripheral blood. PLoS One. 2012; 7, e32350.

9. Ji YH, Ji JL, Sun FY, Zeng YY, He XH, Zhao JX, et al. Quantitative proteomics analysis of chondrogenic differentiation of $\mathrm{C} 3 \mathrm{H} 10 \mathrm{~T} 1 / 2$ mesenchymal stem cells by iTRAQ labeling coupled with on-line two-dimensional LC/MS/MS. Mol Cell Proteomics. 2010;9:550-64.

10. Lee SK, Kim Y, Kim SS, Lee JH, Cho K, Lee SS, et al. Differential expression of cell surface proteins in human bone marrow mesenchymal stem cells cultured with or without basic fibroblast growth factor containing medium. Proteomics. 2009;9:4389-405.

11. Sun HJ, Bahk YY, Choi YR, Shim JH, Han SH, Lee JW. A proteomic analysis during serial subculture and osteogenic differentiation of human mesenchymal stem cell. J Orthop Res. 2006;24:2059-71.

12. Celebi $B$, Elcin AE, Elcin YM. Proteome analysis of rat bone marrow mesenchymal stem cell differentiation. J Proteome Res. 2010;9:5217-27.

13. Welsh Gl, Griffiths MR, Webster KJ, Page MJ, Tavare JM. Proteome analysis of adipogenesis. Proteomics. 2004;4:1042-51.

14. Celebi B, Elcin YM. Proteome analysis of rat bone marrow mesenchymal stem cell subcultures. J Proteome Res. 2009;8:2164-72.

15. Gene Ontology Consortium. http://www.geneontology.org. Accessed 2 Jan 2014.

16. KEGG: Kyoto Encyclopedia of Genes and Genomes. http://www.kegg.jp. Accessed 21 Jan 2014

17. Schneider RK, Puellen A, Kramann R, Raupach K, Bornemann J, Knuechel R, et al. The osteogenic differentiation of adult bone marrow and perinatal umbilical mesenchymal stem cells and matrix remodelling in threedimensional collagen scaffolds. Biomaterials. 2010;31:467-80.

18. Han I, Jeong SJ, Lee HJ, Koh W, Lee HJ, Lee EO, et al. Proteomic analysis of mesenchymal stem-like cells derived from ovarian teratoma: potential role of glutathione S-transferase M2 in ovarian teratoma. Proteomics. 2011;11:352-60.

19. Kurpinski K, Lam H, Chu J, Wang A, Kim A, Tsay E, et al. Transforming growth factor-beta and notch signaling mediate stem cell differentiation into smooth muscle cells. Stem Cells. 2010;28:734-42.

20. Dave JM, Bayless KJ. Vimentin as an integral regulator of cell adhesion and endothelial sprouting. Microcirculation. 2014;21:333-44.
21. Eckes B, Colucci-Guyon E, Smola H, Nodder S, Babinet C, Krieg T, et al. Impaired wound healing in embryonic and adult mice lacking vimentin. J Cell Sci. 2000;113:2455-62.

22. Rogel MR, Soni PN, Troken JR, Sitikov A, Trejo HE, Ridge KM. Vimentin is sufficient and required for wound repair and remodeling in alveolar epithelial cells. FASEB J. 2011;25:3873-83.

23. Nieminen M, Henttinen T, Merinen M, Marttila-Ichihara F, Eriksson JE, Jalkanen S. Vimentin function in lymphocyte adhesion and transcellular migration. Nat Cell Biol. 2006;8:156-62.

24. Andreolas C, Kalogeropoulou M, Voulgari A, Pintzas A. Fra-1 regulates vimentin during $\mathrm{Ha}$-RAS-induced epithelial mesenchymal transition in human colon carcinoma cells. Int J Cancer. 2008;122:1745-56.

25. Kim KR, Choi HN, Lee HJ, Baek HA, Park HS, Jang KY, et al. A peroxisome proliferator-activated receptor gamma antagonist induces vimentin cleavage and inhibits invasion in high-grade hepatocellular carcinoma. Oncol Rep. 2007;18:825-32.

26. Burch TC, Watson MT, Nyalwidhe JO. Variable metastatic potentials correlate with differential plectin and vimentin expression in syngeneic androgen independent prostate cancer cells. PLoS One. 2013;8, e65005.

27. Borges BE, Teixeira VR, Appel MH, Steclan CA, Rigo F, Filipak Neto F, et al. De novo galectin-3 expression influences the response of melanoma cells to isatin-Schiff base copper (II) complex-induced oxidative stimulus. Chem Biol Interact. 2013;206:37-46.

28. Margadant C, van den Bout I, van Boxtel AL, Thijssen VL, Sonnenberg A. Epigenetic regulation of galectin-3 expression by beta1 integrins promotes cell adhesion and migration. J Biol Chem. 2012;287:44684-93.

29. Saravanan C, Liu FT, Gipson IK, Panjwani N. Galectin-3 promotes lamellipodia formation in epithelial cells by interacting with complex N-glycans on alpha3beta1 integrin. J Cell Sci. 2009;122:3684-93.

30. Liu W, Hsu DK, Chen HY, Yang RY, Carraway 3rd KL, Isseroff RR, et al. Galectin-3 regulates intracellular trafficking of EGFR through Alix and promotes keratinocyte migration. J Invest Dermatol. 2012;132:2828-37.

31. Jia W, Kidoya H, Yamakawa D, Naito H, Takakura N. Galectin-3 accelerates M2 macrophage infiltration and angiogenesis in tumors. Am J Pathol. 2013;182:1821-31.

32. Boscher C, Nabi IR. Galectin-3- and phospho-caveolin-1-dependent outsidein integrin signaling mediates the EGF motogenic response in mammary cancer cells. Mol Biol Cell. 2013;24:2134-45.

33. Al-Nedawi KN, Czyz M, Bednarek R, Szemraj J, Swiatkowska M, CierniewskaCieslak A, et al. Thymosin beta 4 induces the synthesis of plasminogen activator inhibitor 1 in cultured endothelial cells and increases its extracellular expression. Blood. 2004;103:1319-24.

34. Freeman $\mathrm{KW}$, Bowman BR, Zetter BR. Regenerative protein thymosin beta-4 is a novel regulator of purinergic signaling. FASEB J. 2011;25:907-15.

35. Cierniewski CS, Malinowski M, Bednarek R, Cierniewska-Cieslak A. Adhesive and proteolytic phenotype of migrating endothelial cells induced by thymosin beta-4. Ann N Y Acad Sci. 2007;1112:123-39.

36. Cha HJ, Philp D, Lee SH, Moon HS, Kleinman HK, Nakamura T. Overexpression of thymosin beta 4 promotes abnormal tooth development and stimulation of hair growth. Int J Dev Biol. 2010;54:135-40.

37. Moon EY, Im YS, Ryu YK, Kang JH. Actin-sequestering protein, thymosin beta-4, is a novel hypoxia responsive regulator. Clin Exp Metastasis. 2010;27:601-9.

38. Badamchian M, Fagarasan MO, Danner RL, Suffredini AF, Damavandy $H$, Goldstein AL. Thymosin beta(4) reduces lethality and down-regulates inflammatory mediators in endotoxin-induced septic shock. Int Immunopharmacol. 2003;3:1225-33.

39. Waanders E, Croes HJ, Maass CN, te Morsche RH, van Geffen HJ, van Krieken $\mathrm{JH}$, et al. Cysts of PRKCSH mutated polycystic liver disease patients lack hepatocystin but express Sec63p. Histochem Cell Biol. 2008;129:301-10.

40. Loo YM, Gale Jr M. Immune signaling by RIG-I-like receptors. Immunity. 2011;34:680-92.

41. Zhang NN, Shen SH, Jiang $\sqcup$, Zhang W, Zhang HX, Sun YP, et al. RIG-I plays a critical role in negatively regulating granulocytic proliferation. Proc Nat Acad Sci U S A. 2008;105:10553-8. 